\title{
Analysis of compaction of railway ballast by different maintenance methods using DEM
}

\author{
Jean-Francois Ferellec ${ }^{1, *}$, Robert Perales ${ }^{2}$, Viet-Hung $\mathrm{Nhu}^{3}$, Michel Wone ${ }^{1}$ and Gilles Saussine ${ }^{1}$ \\ ${ }^{1}$ SNCF Reseau, I\&P-LVE, 6 av. F. Mitterrand, 93574 La Plaine St Denis cedex, France \\ ${ }^{2}$ AD'missions, 20 rue Brunel, 75017 Paris, France \\ ${ }^{3}$ Vibrateam, 28 chemin du Petit Bois, 69134 Ecully cedex, France
}

\begin{abstract}
Railway traffic continuously increasing, ballasted tracks need more efficient maintenance processes. Lines with long welded rails which are prone to buckling during heat waves require stabilisation before being fully operational. Stabilisation is performed either naturally using regular traffic at penalising lower speeds, dynamic stabilisation of sleepers or alternatively crib compaction. The objective of this paper is to apply the NSCD approach of DEM to simulate the processes of dynamic stabilisation and crib compaction as they are realised on site and compare their performance in terms of ballast compaction and lateral resistance. The results showed that NSCD is perfectly appropriate to simulate these maintenance processes and estimate their performance.
\end{abstract}

\section{Introduction}

As railway traffic of passengers and freight is continuously increasing, maintenance of ballasted tracks becomes more and more demanding particularly in the case of tracks using long welded rails prone to buckling during heat waves. To avoid this kind of incident, it is crucial to keep the lateral resistance of the track above a critical level. After their geometrical realignment by the process of tamping which loosens the ballast, tracks require stabilisation to achieve a high level of lateral resistance.

The simplest stabilisation method, called natural stabilisation, consists in successive passages of regular traffic trains at lower speed which is equivalent to applying cyclic vertical loads to the sleepers for a given time. This situation is equivalent to a dynamic uniaxial loading test on a sample. This natural stabilisation is progressively replaced by faster operations which do not disturb the regular train traffic. The most popular alternative method is the dynamic stabilisation where the rails are vibrated laterally while applying a vertical load on them using dedicated equipment. These conditions are typical of a biaxial loading test with varying confining pressure. The second option, called crib compaction and relatively new in railway maintenance, is the direct vertical packing of the ballast located between the sleepers and in the shoulders of the track. This approach is performed by a set of plates of different sizes and locations vibrating vertically while applying a vertical load. This case is similar to the compaction of a free surface granular medium.

In order to avoid in situ campaigns costly in terms of time and budget, both alternative stabilisation processes were analysed numerically by using the discrete element method (DEM) which models granular materials, like ballast, as objects interacting through contacts laws [1]. The code LMGC90 based on the non-smooth contact dynamics or NSCD approach of DEM was used in this study [2]. The aim of this study is to compare their performances in terms of ballast compaction and lateral resistance of sleepers.

The first section briefly presents the NSCD approach of DEM. The second one describes the simulations conditions. The third part shows a comparison of ballast compaction and lateral resistance obtained after using both maintenance processes.

\section{NSCD approach of DEM}

In conventional DEM, a granular material is considered as an assembly of objects interacting through a contact law [1], implementing explicitly the mechanical interaction using an elastic force depending on the overlap between these objects, a sliding criterion limiting the tangential force at contact and potentially a damping force function of the overlapping speed. The calculation cycle in this model consists of: first the detection of the contacts between the objects; second the calculation of the contact forces from the overlaps between them; third the calculation of their displacements by a double integration of their acceleration on a time interval called time step using Newton's Law. The displacements will lead to the update of the positions of the objects ready for another cycle. In this conventional DEM, the most common object is the sphere because it makes the contact detection step easier. However spheres reproduce

Corresponding author: jean-francois.ferellec@reseau.sncf.fr 
the behaviour of a granular material only in a qualitative way. Different approaches were developed to achieve results more quantitatively reliable but they were not really effective in capturing the irregular shape of real particles [3-7]. It is only recently that some models were more successful in reproducing real particles shape by using arrangement of spheres and particularly ballast stones [8-13].

The Non-Smooth Contact Dynamic (NSCD) is a particular approach of DEM, based on implicit time integration and non-smooth formulation of mutual exclusion and dry friction between objects [2, 14-16]. The contact law is defined by a non-smooth relation between the normal force and the normal relative velocity and Signorini conditions. A restitution coefficient relating the relative velocities before and after contact can be introduced in the contact law. The normal and tangential coefficients of restitution can be set equal to zero to reflect the total dissipation of kinetic energy of a particle into the dense packing of particles of ballast. The NSCD code called LMGC90 which is capable of modelling a collection of rigid particles of polyhedral shape has been used in the present study.

\section{Conditions of simulations}

Both dynamic stabilisation and crib compaction were simulated using a four-sleeper track section (fig.1) with ballast particles represented by 90,000 rigid polyhedrons constructed from scans of real ballast stones following French railway ballast requirements. It presents a slope on one side and a vertical wall on the other side as if a contiguous line was present. The friction coefficient between particles and between particles and sleepers has been determined equal to 0.8 , the ballast stone density to $2700 \mathrm{~kg} / \mathrm{m}^{3}$ and the time step to $2 \times 10^{-4} \mathrm{~s}$ [17]. The sample is prepared by generating the ballast particles above the surface and letting them settle under gravity using a reduced friction to achieve the desired apparent density. Before stabilisation, the track goes through a process called tamping [18] which consists of lifting the sleepers and then squeezing the ballast underneath them using the tines which are represented in figure 1 .

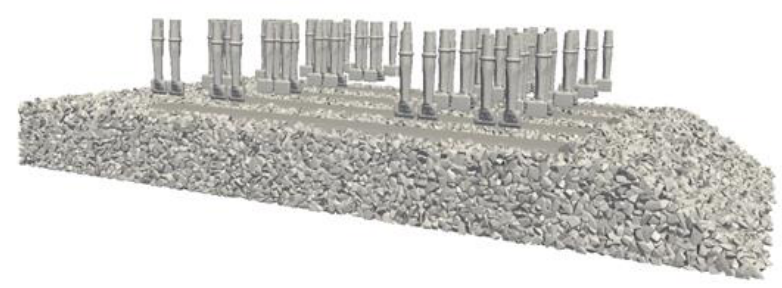

Fig. 1. Four-sleeper configuration model with tamping tines

Figure 2 shows how the six compacting plates are positioned during the crib compaction process: four between the sleepers and two at their ends. They compact the ballast by vibrating vertically during $2 \mathrm{~s}$ at $50 \mathrm{~Hz}$ with a load varying between 5 and $45 \mathrm{kN}$, values which are typical for this process. The six-plate compactor is sequentially moved between the sleepers. In order to keep similar conditions for comparison, the analysis focused on the inside sleepers and cribs and hence only the inside sleepers were subjected to dynamic stabilisation. The dynamic stabilisation is processed in the simulations by exciting the inside sleepers with a signal based on the specifications of the stabilising equipment. The lateral velocity of the sleepers is oscillated using an amplitude varying in a sinusoidal way which corresponds to the displacement of the stabilising equipment along the track. Figure 3.a shows two vibration waves representing the passage of two successive stabilising equipments as seen by each sleeper. Simultaneously two sinusoidal shaped vertical loads are applied (Figure 3.b). In this study, the vibration signal has a frequency of $25 \mathrm{~Hz}$, a maximum amplitude of $2.5 \mathrm{~mm}$ and a rolling speed of $2000 \mathrm{~m} / \mathrm{s}$, values which are typical for this process. Finally the lateral resistance of the track is determined by applying a progressive load laterally on both inside sleepers and measuring simultaneously their lateral displacement.

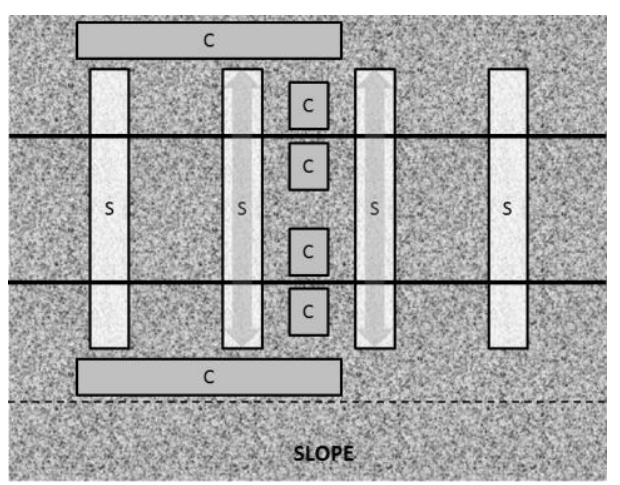

Fig. 2. Sample with sleepers (S) and compactor plates (C)
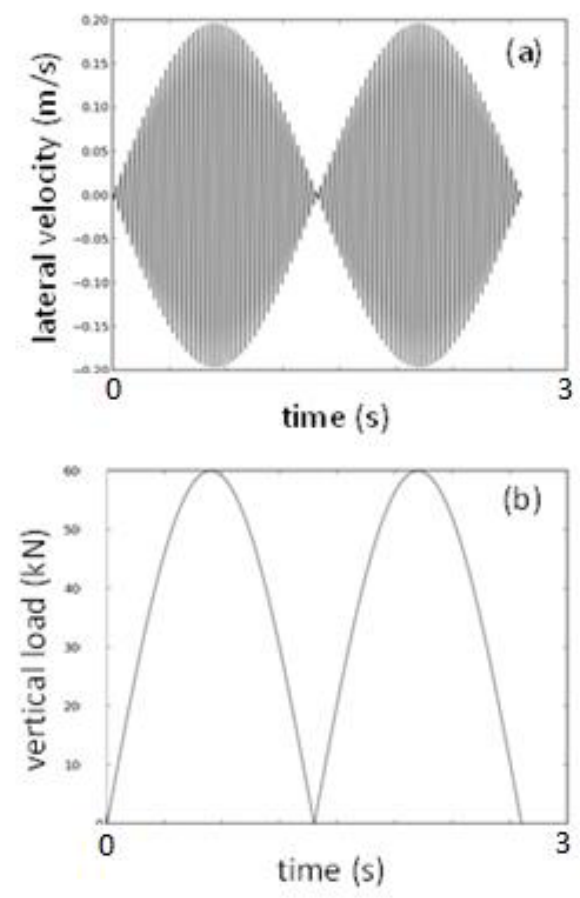

Fig. 3. Lateral velocity (a) and load (b) of dynamic stabilisation 


\section{Comparison of methods}

Both processes of dynamic stabilisation and crib compaction were compared in terms of solid fraction gain under the sleepers and in the cribs (between sleepers). Figure 4 shows the evolution of the solid fraction for the ballast under the inside sleepers for both processes. The dynamic stabilisation lasts a little bit less than $3 \mathrm{~s}$ while the crib compaction lasts $8 \mathrm{~s}$ for the whole sample. Regarding the dynamic stabilisation, the effect of two waves is clearly observed, the second bringing less compaction. The ballast under the inside sleepers reach almost the same final solid fraction of $61.3 \%$, even if they start with different initial values, homogenising the ballast density among the sleepers. The crib compaction seems to be linear with time. The black bottom line of figure 4 shows that the solid fraction under the first sleeper starts to increase during the $2 \mathrm{~s}$ crib compaction on one side of the sleeper and the other one on the other side offering a solid fraction gain of more than $1 \%$. The second sleeper obtains the same gain thus leading to a different final value. It does not seem to homogenise the solid fraction under the sleepers as the dynamic stabilisation which acts directly on the sleepers. This however could potentially be achieved by tuning the vibration frequency or vertical load of the crib compaction process.

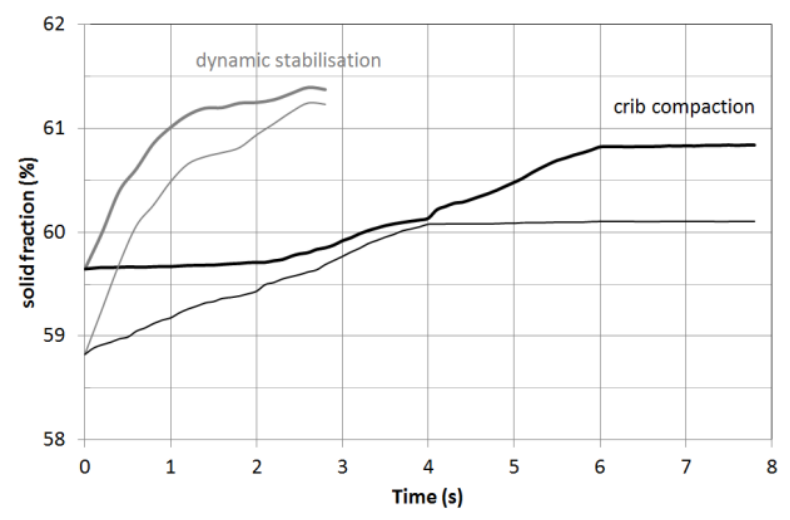

Fig. 4. Comparison of ballast solid fraction under sleepers for dynamic stabilisation (grey) and crib compaction (black)

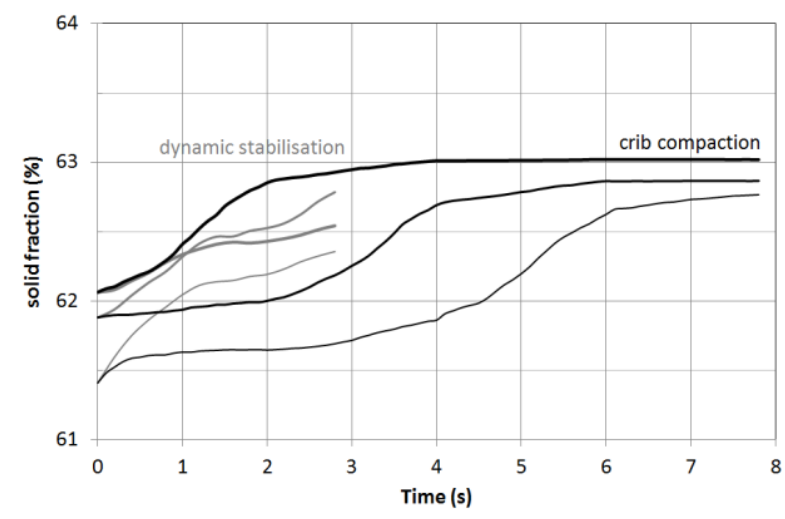

Fig. 5. Comparison of ballast solid fraction in cribs for dynamic stabilisation (grey) and crib compaction (black)
Figure 5 shows the evolution of ballast solid fraction in all three cribs along the inside sleepers under sleeper bottom level for both processes. The effect of the crib compaction process is clearly observable. The solid fraction increases during $2 \mathrm{~s}$ for all cribs sequentially as expected reaching in the end almost the same final value. The dynamic stabilisation also increases the solid fraction in cribs but in a lesser way which is understandable as cribs are not directly vibrated.

Figure 6 shows the initial and final ballast solid fractions obtained along the track components for both processes. Both processes significantly compact the ballast under the sleepers although dynamic stabilisation seems more efficient. In cribs, the opposite effect is observed. Initially quite heterogeneous along the track, the ballast density presents a more homogeneous profile after dynamic stabilisation. The crib compaction seems to give the same gain to all areas as the ' $\mathrm{W}$ ' shaped density profile along the track is almost unchanged.

Globally, in terms of ballast compaction, dynamic stabilisation seems more efficient than crib compaction using the parameters stated above which are typical of actual operations. Different sets of crib compaction parameters could lead to different final compaction levels inside the ballast.

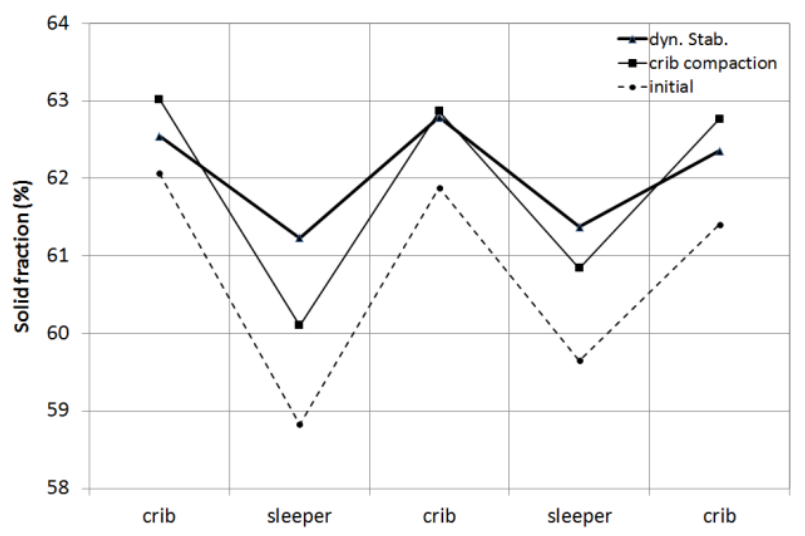

Fig. 6. Comparison of final solid fraction under sleepers and cribs

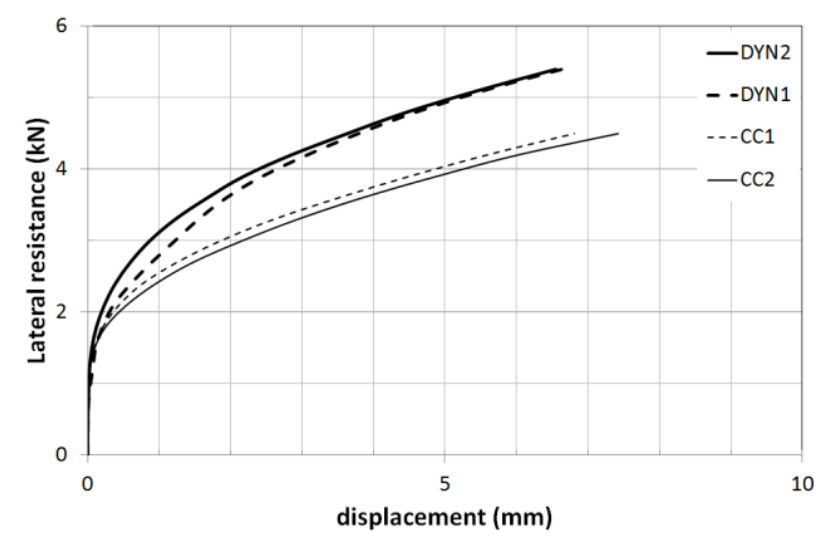

Fig. 7. Lateral resistance as a function of sleeper displacement for inside sleepers $(1,2)$ for dynamic stabilisation (DYN) and crib compaction (CC) 
In terms of lateral resistance, dynamic stabilisation seems to give slightly better results. Figure 7 shows the lateral resistance obtained for the inside sleepers for both processes. For each process, both sleepers (identified as 1 and 2) present very similar lateral resistance. The difference of resistance between both processes seems to be around $1 \mathrm{kN}$ at $5 \mathrm{~mm}$ displacement meaning that lateral resistance obtained after crib compaction is just 20\% lower than the one after dynamic stabilisation. As an engineering point of view, this makes crib compaction a suitable alternative to dynamic stabilisation. By tuning its operating parameters, it could also be improved. The side effects of this tuning on the global ballast behaviour must also be identified. Dynamic stabilisation can also be improved by tuning its parameters. Previous studies have shown that decreasing the speed of dynamic stabilisation increased compaction [19].

\section{Conclusions}

The NSCD approach of DEM has been used to analyse dynamic stabilisation and crib compaction, two different ballasted tracks maintenance processes, in terms of compaction capabilities and track lateral resistance.

The results showed that NSCD is perfectly adequate for this type of analysis. Its application showed that both stabilisation methods fulfil their intended purpose of densifying the ballast under sleepers and increasing the lateral resistance of the track although dynamic stabilisation achieves it in a better way.

On the point of view of railway engineering, it shows that crib compaction is an adequate potential alternative to dynamic stabilisation even if it is slightly less efficient. This limitation in performance could be addressed by tuning its parameters, particularly the vibration frequency or vertical load.

NSCD is presently used to realise an impact study regarding parameters of both maintenance operations in order to optimise them. A deeper analysis of the effect of the parameters on various aspects of the phenomena occurring at the scale of the ballast stones will be realised.

Another study identifying the contribution of the different components of ballasted tracks to lateral resistance has been started. It includes the type of sleeper and the shoulder size among others.

\section{References}

1. P.A. Cundall, and O.D.L. Strack, Geotechnique, 29, 1, 47-65 (1979)

2. J.J. Moreau, European J. Mech. A Solids, 13, 4, suppl., 93-114 (1994)

3. J.R. Williams, and A.P. Pentland, Eng. Comput., 9, 115-127 (1992)

4. X. Lin, and T.T. Ng, Geotechnique, 47, 2, 319-329, 1997.

5. G.G.W. Mustoe, and M. Miyata, J. Eng. Mech., 127, 10, 1017-1026 (2001)

6. P.W. Cleary, Engineering Computations, 21, 2-4, 169-204 (2004)
7. L. Pournin, Granular Matter, 7, 2-3, 119-126 (2005)

8. R. Hart, P.A. Cundall, and J. Lemos, Int. J. Rock Mech. Min. Sci. Geomech. Abstr., 25, 3, 117-125 (1988)

9. L.B. Wang, J.Y. Park, and Y.R. Fu, Construction and Building Materials, 21, 2, 338-346 (2007)

10. M. Price, V. Murariu, and G. Morrison, Proceedings of Discrete Element Methods Conference, Brisbane, Australia (2007)

11. Y. Lee, Granular Matter, 11, 5, 307-315 (2009)

12. J.-F. Ferellec and G.R. McDowell, Granular Matter, 12, 5, 459-467 (2010)

13. J.-F. Ferellec, and G.R. McDowell, Geotechnique, 60, 3, 227-232 (2010)

14. J.J. Moreau, European J. Mech. A Solids, 13, 4, suppl., 93-114 (1994)

15. G. Saussine, C. Cholet, P.E. Gautier, F. Dubois, C. Bohatier, J.J. Moreau, Comput. Methods Appl. Mech. Eng., 195, 2841-2859 (2006)

16. F. Dubois and M. Renouf, Proceedings of Multibody 2007, Politecnico di Milano, Milano, Italy (2007)

17. G. Saussine, Contribution to the modelling of tridimensional granular materials: application to ballast, PhD thesis (2004)

18. J.-F. Ferellec, R. Perales, P.-E. Laurens, M. Woné, J. Plu and G. Saussine, Proceedings of IV International conference on Particle-based Methods - Fundamental and Applications, Particles 2015, Barcelona, Spain (2015)

19. J.-F. Ferellec, M. Woné, R. Perales and G. Saussine, Proceedings of III International conference on Railway Technology: Research, Development and Maintenance, Cagliari, Italy (2016) 